\section{Locally enhanced chemotherapy by electroporation: clinical experiences and perspective of use of electrochemotherapy}

\author{
Ruggero Cadossi', Mattia Ronchetti ${ }^{1} \&$ Matteo Cadossi ${ }^{*, 2}$
}

\begin{abstract}
Electroporation is used to enhance drug diffusion and gene delivery into the cytosol. The combination of electroporation and cytotoxic drugs, electrochemotherapy $(E C T)$, is used to treat metastatic tumor nodules located at the skin and subcutaneous tissue. The objective response rate following a single session of treatment exceeds $80 \%$, with minimal toxicity for the patients. The efficacy of ECT in the bone and visceral metastasis is currently investigated, and Phase II studies have been completed. ECT has been used to treat skin primary tumors, except melanoma, and is under investigation for locally advanced pancreatic cancer. Early evidence suggests that treatment of tumor nodules with ECT recruits components of the immune system and eliciting a systemic immune response against cancer is a challenging clinical perspective. Considering the proven safety in several different clinical applications electroporation should be viewed as a clinical platform technology with wide perspectives for use in ECT, gene therapy and DNA vaccination.
\end{abstract}

\section{Electrochemotherapy}

Electrochemotherapy (ECT) is a combination treatment that exploits the administration of chemotherapeutic drugs in association to cell membrane electroporation (EP) [1]. In literature, EP and electropermeabilization are terminologies both used to indicate structural changes in the cell membrane lipid bilayer induced by local electric field pulses; throughout this review the term EP and ECT will be used as they have been routinely adopted in clinical practice. EP of the cell membrane occurs when electric field pulses are applied to cells or biological tissue; EP depends on cell characteristics (shape, size, cytoskeleton structure and membrane composition) and on electrical parameters (amplitude and duration of electric pulses, number of pulse and repetition frequency). At the cell membrane level, EP occurs when an external electric field above the threshold value of the transmembrane potential (approximately 1.5 V) is applied. The molecular mechanism(s) of cell membrane EP has not been fully explained; models proposing pore formation posit that pores are unstable, they are created rapidly (approximately $10^{-6} \mathrm{~s}$ ) and disappear within a few seconds to several minutes after exposure to the electric field $[2,3]$. Recent molecular dynamic simulations have shed additional insight into the mechanism of lipid bilayers permeabilization demonstrating water diffusion within the membrane structure [4]. Cell membrane EP finally allows the transport of hydrophilic molecules across the plasma membrane.

EP increases permeability of the cell membrane to agents that, normally, can scarcely penetrate into the cells. Hydrophilic molecules or large molecules like DNA and RNA, albeit through different processes, can enter into the cytosol of cells as a result of EP. The importance of EP and its foreseen clinical applications were immediately perceived by researchers who envisaged its use for gene and

'Clinical Research, IGEA SpA, Via Parmenide 10/A, Carpi, Italy

${ }^{2}$ Istituto Ortopedico Rizzoli, University of Bologna, Bologna, Italy

*Author for correspondence: Tel.: +39 059 699600; Fax: +39 059 695778; r.cadossi@igeamedical.com

\section{KEYWORDS}

- bleomycin • cancer

- cisplatin $\bullet$ Cliniporator ${ }^{\mathrm{TM}}$

- electrochemotherapy

- electroporation • ESOPE

- gene therapy $\bullet$ melanoma

- metastases 
drug electrotransfer: electrogenetherapy [5] and ECT, respectively $[1]$.

The most important electrical parameters to be controlled/defined for cell membrane EP are: amplitude and duration of the electric pulse, number of pulses and pulse frequency. Nevertheless, homogenous and complete EP of all cells in a tissue requires the calculation of electric field distribution in the tissue based on the geometry of the electrode used and tissue specific electrical properties. EP efficiency is also affected by the size of the target cells in the tissue: small cells require exposure to higher electric filed to obtain cell membrane EP [6,7]. In EP-mediated gene transfer to cells in tumors, transfection efficiency is also influenced by composition of the extracellular matrix: soft tumors with larger spherical cells, low proteoglycan and collagen content, and low cell density are more effectively transfected [8]. However, the presence of a mineralized matrix does not prevent cell membrane EP [9].

In the perspective of tumor treatment, preclinical studies have been extensively conducted first by Lluis Mir and, later, by Gregor Sersa, who identified the optimal electric field characteristics and the appropriate neoplastic drugs whose cytotoxic activity could be potentiated by combination with cell membrane EP. Among several drugs that have been evaluated in association to EP, bleomycin and cisplatin cytotoxicity was significantly augmented: in vitro bleomycin cytotoxicity increased up to 1000 -fold and cisplatin by 80 -fold [1,10]. These observations date back to the end of the 1980s (Figure 1) [1,6]. EP, in combination with more recently developed drugs, for example, small tyrosine kinase inhibitors, has not yet been investigated. On the other hand, only recently, EP efficiency of different protocols of electric field pulses have been tested [11]. In vivo preclinical studies readily supported the in vitro observations showing that a single ECT treatment could heal tumors transplanted in mice. Furthermore, it was demonstrated that ECT was effective in different types of tumor transplanted [12-18]. Recently, experimental bone metastases in rats have been successfully treated by ECT, providing the necessary preclinical evidence for a Phase II study in patients [19]. ECT has also been tested in CNS experimental metastases in rats; on the bases of the study results, a Phase I study has been designed and the first patient has been treated without side effects [20,21].

Interestingly, it has been reported that in nude mice, ECT treatment is less effective compared with immune competent mice. This observation suggests a role for the immune system in the response of tumor nodules to ECT [22-25]. These observations are particularly relevant as recent data suggest the involvement of the immune system in patients too $[26,27]$.

In vitro and in vivo investigation of the combination of cell membrane EP and properly selected chemotherapeutic agents set the foundation for the translation of ECT to clinical practice standardizing the use of bleomycin (usually administered systemically, i.e., intravenously) and cisplatin (administered topically into the tumor). Even when bleomycin is administered intravenously, the increased cytotoxic effect of the drug is limited to the area exposed to the electric field pulses that induce membrane EP; a sort of locally enhanced chemotherapy.

In the 1990s, early clinical experiences were conducted with ECT [28-30]. The EP protocol foresaw to expose the tumor nodule to eight pulses of 1000-1300 V of amplitude per cm of separation between electrodes with each pulse lasting $100 \mu$ s. Initial treatments were conducted using intratumoral bleomycin at a dosage of approximately $0.5 \mathrm{U}(500 \mathrm{IU})$ per $100 \mathrm{~mm}^{3}$ of tumor. Prospective randomized studies, drug versus drug plus EP, clearly showed that the combined treatment was more effective compared with the drug alone [31-35]. Studies were conducted in patients with metastatic tumor nodules at the skin or subcutaneous tissue; the end point of the studies was tumor volume change according to the WHO criteria [36]:

- Complete response: disappearance of tumor nodule

- Partial response: tumor nodule reduction by more than $50 \%$

- No change: tumor nodule reduction by less than $50 \%$

- Progressive disease: tumor volume increase

- Objective response: complete response plus partial response

Data gathered during the monitored trials demonstrated that the percentage of patients attaining an objective response (i.e., complete response plus partial response) was significantly higher in the group of patients receiving ECT 


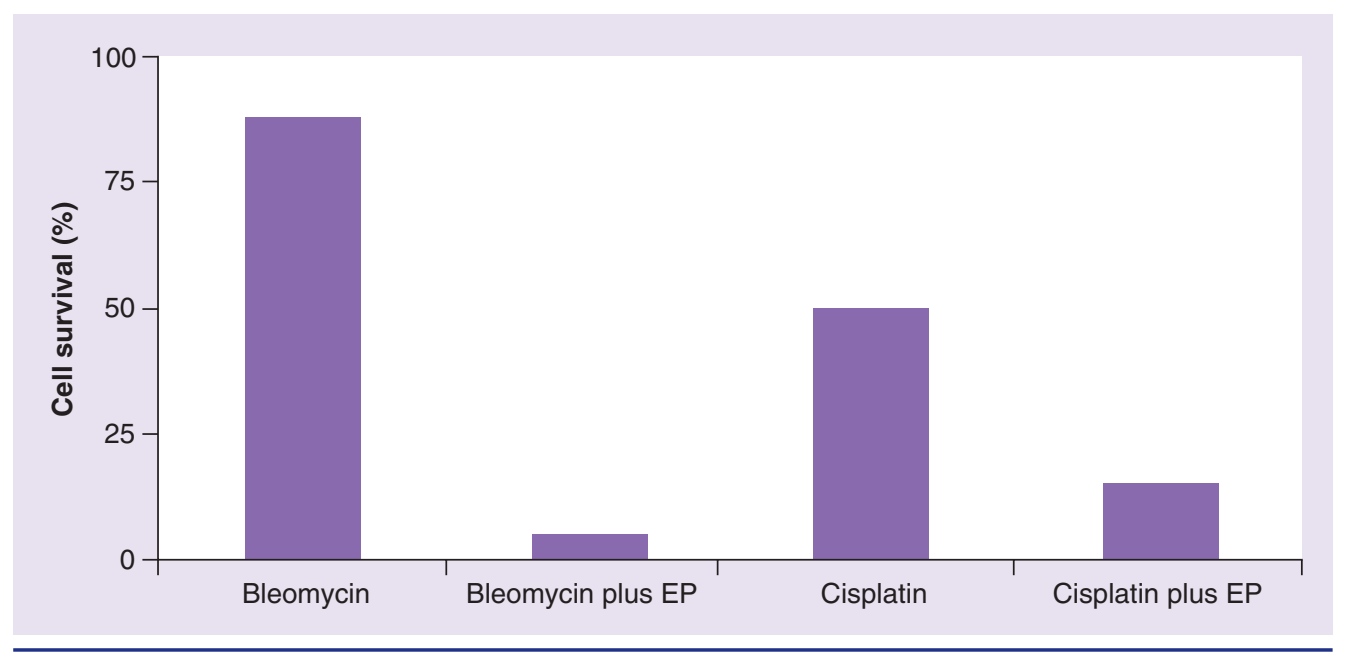

Figure 1. DCF3 cell survival cultured in the presence of bleomycin $10^{-8} \mathrm{M}$ or cisplatin $15 \mu \mathrm{M}$ and exposed to electroporation.

EP: Electroporation.

Data taken from $[1,10]$.

in comparison with the group treated with bleomycin or cisplatin alone. The mean objective response rate was $84.8 \%$ in patients receiving ECT and $24.8 \%$ in those treated with bleomycin or cisplatin alone (Table 1).

In addition, it was demonstrated that ECT resulted in a statistically significantly higher local control rate after 2 years (Table 2) [33,37]. In patients treated with ECT, no local relapses were observed in patients when a complete response was first achieved. Health-related quality of life was not evaluated in controlled clinical trials.

In accordance with preclinical experiences, in patients ECT treatment was effective in different tumor histotypes, albeit with slight variations in objective response (OR) rates [39-43]. This observation has been repeatedly confirmed and it converses from current oncology guidelines (i.e., biological drugs and targeted therapies), nevertheless it can explained by considering the biophysical mechanism, EP, at the bases of ECT treatment that allows the direct diffusion of the cytotoxic drug to the cytoplasm and to DNA [1].

\section{- Development of standard operating procedures}

Although ECT efficacy was well demonstrated, differences among treatment protocols, the lack of defined operating procedures and the use of different pulse generators prevented the widespread adoption of ECT in the clinical practice. In response to this need the European Commission funded two projects:
Cliniporator $^{\mathrm{TM}}$ (IGEA, SpA, Italy) in 2000 for the development of a medical grade device for EP and, in 2003, the international multicenter clinical study ESOPE, which supported the validation and adoption of ECT. The results of the ESOPE study were collected and published in 2006 in a supplemental issue of the European Journal of Cancer [44]. Standard operating procedures (SOPs) [45] for ECT using the Cliniporator device were published along with the results of the ESOPE study; within this project the indications for use, the route of administration of cytotoxic agent and the type of anesthesia to be applied were defined [45].

According to the ESOPE SOPs, bleomycin should be administered intravenously $\left(15,000 \mathrm{IU} / \mathrm{m}^{2}\right.$ in a bolus in $\left.30-60 \mathrm{~s}\right)$ or locally through intratumoral injection. Intratumoral bleomycin should be administered in the form of solution $(1000 \mathrm{IU} / \mathrm{ml})$, in a dose of $1000 \mathrm{IU} / \mathrm{cm}^{3}$ in lesions $<0.5 \mathrm{~cm}^{3}, 500 \mathrm{IU} / \mathrm{cm}^{3}$ in lesions $\geq 0.5 \mathrm{~cm}^{3}$ and $\leq 1 \mathrm{~cm}^{3}$, and $250 \mathrm{IU} / \mathrm{cm}^{3}$ in lesions $>1 \mathrm{~cm}^{3}$. Cisplatin $(2 \mathrm{mg} / \mathrm{ml})$ is only administered intratumoral. The dose of cisplatin also depends on the size of neoplastic lesions. Tumors larger than $1 \mathrm{~cm}^{3}$ should be treated with a dose of $0.5 \mathrm{mg} / \mathrm{cm}^{3}$, those $\geq 0.5 \mathrm{~cm}^{3}$ and $\leq 1 \mathrm{~cm}^{3}$ should be treated with a dose of $1 \mathrm{mg} / \mathrm{cm}^{3}$, and in lesions smaller than $0.5 \mathrm{~cm}^{3}$, a dose of $2 \mathrm{mg} / \mathrm{cm}^{3}$ should be applied. One of the inclusion criteria of the ESOPE study was a maximum lesion size of $\leq 3 \mathrm{~cm}$. Within 8-28 min after intravenous administration of bleomycin, EP of the tumor 


\begin{tabular}{|c|c|c|c|c|}
\hline Study (year) & Drug & Patients (n)/histotype & $\begin{array}{l}\text { Lesion OR rate, drug } \\
\text { vs drug plus EP (\%) }\end{array}$ & Ref. \\
\hline Glass et al. (1996) & it. bleomycin & 5/melanoma & 0 vs 95 & [31] \\
\hline Heller et al. (1998) & it. bleomycin & $\begin{array}{l}\text { 34/melanoma, Kaposi, } \\
\text { BCC, SCC }\end{array}$ & 0 vs 91 & {$[32]$} \\
\hline Sersa et al. (2000) & it. cisplatin & 10/melanoma & 38 vs 78 & {$[33]$} \\
\hline Byrne et al. (2005) & it. bleomycin & 19/melanoma & 32 vs 78 & [34] \\
\hline Gaudy et al. (2006) & it. bleomycin & 12/melanoma & 54 vs 82 & [35] \\
\hline
\end{tabular}

nodules should be completed. Following intratumoral administration of bleomycin or cisplatin, EP should be completed within $10 \mathrm{~min}$ of drug injection. The physician should make sure that electric pulses are applied to the whole tumor volume to ensure all cells are electroporated. In order to minimize the pain associated with the delivery of electric pulses, the procedure should be performed under appropriate anesthesia care. Local anesthesia should be achieved by injection of lidocaine (2\%) and adrenaline $(0.5 \%)$ around the area of the treated lesion; care should be taken not to exceed the maximum dose of the anesthetic agent, especially in patients with multiple lesions to be treated. Tumescent local anesthesia has been recently used for cutaneous metastases [46]. General anesthesia should be considered when treating larger or numerous lesions. Complementary therapy to chemotherapy may include: antiemetics (e.g., ondansetron), corticosteroids (e.g., dexamethasone), paracetamol, NSAIDs and allopurinol.

In most of the cases the procedure is performed in outpatient settings or in 1-day hospitalization. If the lesions inadequately respond to treatment or new lesions develop, ECT may be repeated.

Technological aspects are important too. Pulse protocols and amplitude of the electric pulses have been calculated according to electrode geometry and separation. Square shaped electric pulses are used and the desired pulse amplitude should be maintained through the entire pulse duration. Precise control of EP parameters, obtained by an appropriate design of devices, allows the application of lower voltages with less discomfort for the patient while maintaining high local efficacy of the ECT treatment. In clinical practice, monopolar, direct current, short and intense electric pulses are used. ECT protocols use eight square pulses, each lasting $100 \mu$ s delivered at a frequency of 1 or $5000 \mathrm{~Hz}$. Pulses are delivered through diposable needle or plate electrodes and the electric field applied to each electrode couple is calculated based on electrode separation so that local electric field in the tumor is $400 \mathrm{~V} / \mathrm{cm}$; electrode-specific pulse parameters are automatically set by the Cliniporator. Finally, The electric pulse generator should measure, in real time, the amplitude of the electrical current in the tissue to prevent excess current for patient safety. When electrical pulses are applied in the proximity of the heart electric pulse delivery should occur only during the absolute refractory period. In case of superficial neoplastic lesions, plate electrodes are used. Needle electrodes should be used when treating larger lesions up to $3 \mathrm{~cm}$ deep. Linear configuration needle electrodes are used in treatment of small nodules, while hexagonal configuration needle electrodes are employed in the treatment of larger tumors. Finger electrodes display needles, which are installed on a special thimble-shaped cap to be put on surgeon's finger, are to be used in treatment of lesions located in scarcely accessible anatomical locations (e.g., in the oral cavity).

In a recent meta-analysis, Mali and colleagues observed differences in OR rate among studies conducted before (OR: $77.4 \%$ ) or after the adoption of ESOPE operating procedures

\begin{tabular}{|c|c|c|c|c|c|c|c|}
\hline Study (year) & End point & Tumor & Drug & Follow-up & ЕCT (\%) & Control (\%) & Ref. \\
\hline $\begin{array}{l}\text { Quaglino } \\
\text { et al. (2010) }\end{array}$ & $\begin{array}{l}\text { Local } \\
\text { control }\end{array}$ & Melanoma & Bleomycin & 2 years & 77.0 & None & [38] \\
\hline $\begin{array}{l}\text { Sersa et al. } \\
(2000)\end{array}$ & $\begin{array}{l}\text { Local } \\
\text { control }\end{array}$ & Melanoma & Cisplatin & 2 years & 79.4 & 19 & [33] \\
\hline
\end{tabular}


(OR: 87.8\%) [47]. These results cannot be explained by differences in the drug chemistry, but rather argue in favor of drug administration route and dosage, of the technology used to control and deliver electrical pulses of effective amplitude and of the electrodes design. Overall, it should be acknowledge that the efficacy of ECT equally depends on local drug concentration and on EP of all cells in tumor nodule.

The ESOPE SOPs are presently used in over 120 oncological centers in Europe, and more than 5000 patients have been treated. ECT has shown to be safe and effective in studies adopting the SOPs. The use of intravenously bleomycin is the preferred route for drug administration. European experience on ECT treatment of skin metastases is being collected in the INSPECT registry [48]. Based on clinical information available in the literature, in March 2013, NICE released a specific guidance (\#446) for the use of ECT in skin metastasis from melanoma and nonmelanoma tumors [49-51].

\section{Clinical experiences}

Treatment of malignant lesions located at the skin is often a challenge for the physician as they are resistant to most treatments. They are often associated to widespread disease dissemination and the risk of relapse after radiotherapy or chemotherapy is high. In certain cases, lesions at sites other than the skin may be stable and, therefore, physicians may be reluctant to apply additional systemic chemotherapy to treat skin lesions only. Skin metastases and their treatment seldom affect survival, but they may be very critical for the patient owing to their severe impact on the quality of life. Therefore, locally enhanced chemotherapy by ECT can offer a new important and safe opportunity to control skin localization of the tumor and it should always be positioned within the chemotherapy treatment program by the medical oncologist [44].

ECT is used mainly for treating superficial (i.e., skin and subcutaneous) neoplastic lesions regardless of their histology [52-60]. ECT has been applied in the treatment of skin metastases of squamous cell carcinoma, melanoma, basal cell carcinoma, adenocarcinomas (of the breast, salivary glands and kidneys), Kaposi sarcoma, Merkel cell carcinoma, transitional cell carcinoma of the urinary bladder, and head and neck cancer. ECT may be used, when there is no possibility of surgical intervention, or in treatment of lesions resistant to chemotherapy and radiotherapy. The effects of any therapeutic option are relevant, especially in palliative treatment of painful and bleeding lesions; blood loss from ulcerated tumor nodules is blocked immediately following ECT treatment owing to a vascular lock effect associated to the delivery of electrical pulses $[61,62]$. ECT is well tolerated, it allows for immediate recovery, and may be repeated if necessary when treating large tumor nodules. In patients with skin metastases, achievement of local disease control remains the objective of treatment for the rest of the patient's life. Regional and local treatment methods, such as palliative surgery, repeated irradiation, hyperthermia or isolated limb perfusion or infusion, may also be offered to patients with skin metastases in order to achieve adequate symptom control. If these methods are offered, the risk of complications and toxicities should always be thoroughly discussed and potential benefits weighed against potential risks for the patient. Locally enhanced chemotherapy by ECT is a local treatment modality; its benefits include low invasiveness, rapid treatment effect and absence of severe adverse events. ECT may also be offered to elderly patients, in whom the risk of adverse events and toxicity associated with other methods may be increased due to frailty and/or associated comorbidities [63]. ECT is contraindicated in patients that have had allergic reaction to bleomycin or cisplatin (to the corresponding drug), as well as in those, in whom the lifetime cumulative dose of bleomycin exceeds 400,000 IU [44].

Reports from clinical experiences adopting the operating procedures of the ESOPE study were consistent with those of controlled clinical trials. The meta-analysis of Mali et al., which included 44 studies involving 1894 tumors reported an objective nodule objective response rate to ECT of $84 \%$ [47]. Data analysis demonstrated that ECT was more effective $(\mathrm{p}<0.001)$ than cytotoxic drugs, bleomycin or cisplatin, used alone. According to the meta-analysis findings ECT is more effective in sarcoma compared with carcinoma tumor nodules. However, the analysis could not evaluate differences in time to response according to different tumor types. Small tumor nodules $(<3 \mathrm{~cm})$ are easier to treat compared with larger ones with better clinical response to ECT; intratumoral injection of bleomycin is usually preferred treating small solitary tumor nodules $[47,64]$.

In 2009, Campana et al. reported an improvement in patients' quality of life with respect to wound healing and bleeding, esthetic impairment, 
daily activities, social relations and pain control [57]. Differences in quality of life assessed before and after treatment (both 1 and 2 months after ECT) were statistically significant $(\mathrm{p}<0.005)$. Several authors reported that, between 90.2 and $100 \%$ of patients would accept retreatment, if necessary $[39,44,48,57]$.

In 2011, Matthiessen et al. observed reduced exudation from skin lesions after ECT in five out of 12 patients with advanced breast cancer [48], while among patients treated by Campana et al. the number of individuals with ulcerative lesions decreased from 22 to five due to ECT [57]. Four studies reported intensity of pain decreased after ECT in most patients with painful neoplastic lesions [39,44,54,65].

In 2000, Gehl and Geertsen observed bleeding arrest and crust formation as early as a few hours after application of treatment [61]. Bleeding stopped also in patients reported by Kaehler et al. [65], Snoj et al. [66], Gehl and Geertsen [67], and decreased substantially in those observed by Whelan et al. [68]. In patients with ulcerative lesions, complete response to treatment was achieved [61,68-72]. In addition, in patients with painful neoplastic lesions, pain relief was reported after ECT [68,71]. Finally, ECT has also been applied as neoadjuvant treatment prior to surgery in order to reduce the tumor size, which makes easier and less invasive residual tumor nodule removal by surgical intervention [73] .

Early clinical studies were conducted in patients with in-transit metastatic melanoma [31-35]. Following the publication of the ESOPE study results, several additional studies have been conducted since and lead to identification of ECT role in patients with stage III and IV melanoma [66,74-81]. The involvement of patients' immune cells after ECT has suggested to investigated the effect of combining ECT treatment with systemic immunomodulating agents in melanoma patients $[27,82]$. Preliminary results of combining ECT to ipilimumab indicate that ECT may increase the response to ipilimumab [83].

The efficacy of ECT has also been proven for the treatment of other tumors of the skin, in particular, an indication has been identified for Kaposi sarcoma [84-87]. Treatment with ECT of basal cell carcinomas and squamous cell carcinomas localized in the head in elderly patient should be always considered [56,72,88-90]. Positive results of the treatment with ECT in locoregional recurrence of squamous vulvar cancer have recently been reported [91].
Breast cancer metastasis to the skin and subcutaneous tissue are the most frequent manifestation treated with ECT. In most cases, the treatment has been reserved to very advanced cases with large ulcerated bleeding metastatic nodules [48]. It has been observed that early patient treatment would not require multiple treatment session, save the patient discomfort and improve quality of life [92-94], while achieving higher local tumor control rates and longer local progression-free survivals.

An important role has been recognized to ECT in the treatment of head and neck tumors [95-99]. This anatomical site is specifically complex and the systemic treatments have limited efficacy. On the other hand, established local treatments, such as radiation therapy, surgery and photodynamic therapy may be demanding for the patient and may result in function limitations or disfiguration to a point that the patients' quality of life is largely deteriorated and argues against the treatment choice and benefits. In head and neck cancer patients, ECT results in a minimal or nil function impact and leads to healing of treated tumor lesions without damage to the healthy tissues. Two recent studies have shown the usefulness of ECT in head and neck tumors with high success rate and absence of negative side effects $[100,101]$. However, much attention must be paid to intratumoral use of bleomycin in the oral cavity. The toxicity of bleomycin for mucosal tissues is known and can lead to pain, and large areas of necrosis when injected locally. In the USA, a study on head and neck cancer patients using the SECTA protocol, which called for high local concentration of intratumoral bleomycin, was interrupted because of severe adverse events [102].

According to Gargiulo et al., the role of ECT is not limited to palliative treatment, but may also be the definitive treatment in patients with inoperable head and neck cancer, especially in elderly patients $[90,101]$.

Local tumor control was evaluated in two trials in which patients with melanoma took part $[33,38]$. Local tumor control was achieved in 77 and $79.4 \%$ of patients treated with ECT by Sersa et al. [33] and Quaglino et al. [38], respectively. Sersa et al. demonstrated statistically significant differences with respect to the local tumor control rate in favor of the ECT group in comparison with the group receiving cisplatin only ( $\mathrm{p}<0.0001 ;$ Table 2) [33]. 


\section{ECT safety profile}

In all clinical trials adopting the ESOPE protocol (drug concentration and pulse characteristics) ECT was well tolerated by the patients. The most common adverse effects were local, including: muscle contractions during EP, edema or erythema, and local pain during and after the procedure $(21.5 \%)$. No cases of toxicity related to the administration of bleomycin or cisplatin were reported. The safety profile of ECT is favorable, with a limited number of adverse events, mostly moderate and transient. Few serious adverse events reported in the literature were investigated and considered not related to ECT [44].

\section{Treatment of nonsuperficial metastases}

Recently, ECT treatment of metastases to the bone and to the liver has been investigated. In these settings the procedures are complex and new important operational challenges have been identified and will need further investigation to be solved.

Correct spatial positioning of electrodes, usually individual long needles, requires skill and the support of intraoperative imaging: $x$-ray, ultrasound or computed tomography imaging. Specific instruments have been developed to guide needle insertion and positioning and to guarantee the complete and homogenous coverage of the tumor volume by the applied electric field. The drawback of incorrect electrode positioning may be insufficient EP in the target tissue and may lead to areas of nonelectroporated cells, thus increasing the risk of partial responses and local recurrences. Treatment needs to be carefully planned with dedicated software that indicates where to position electrodes and the required EP pulse conditions (Figure 2) [103,104].
Another issue identified during early-phase clinical trials is represented by monitoring the effect of ECT; while in superficial nodules the visual inspection is sufficient to follow nodule response, for deep seated metastases the use of imaging is mandatory. Nevertheless, the healing process is different from thermal ablation technologies inducing tissue necrosis, thus image interpretation and time interval for repeated examinations need to be that they are performed using computed tomography, MRI or computed tomography/PET. Finally, the treatment of metastases localized to the abdomen, and specifically to the left lobe of the liver, require to synchronize the pulse deliver to the heart absolute refractory period to avoid interference with the heart electrical activity [105-107]. Two earlyphase clinical trials have been completed for the treatment of metastases of different origin to the bone (Phase II study) [108], and synchronous and metachronous metastases from colon cancer localized to the liver (Phase I/II) [109]. The studies' results show that: the treatment of metastases localized in nonsuperficial organs is feasible and safe; ECT has induced resolution or regression of tumor nodules; and patients experienced pain relief when present and improvement of quality of life.

The tuning of the technique and the demonstration of safety and activity of ECT in these experiences will allow appropriate development of Phase II multicenter studies designed to assess ECT efficacy in the different patients populations [Mercuri M et al., Unpublished Data; Gadzijev EM et AL., Unpublished Data]. The road to the use of ECT in nonsuperficial metastases is now open, future studies should investigate the ECT effects
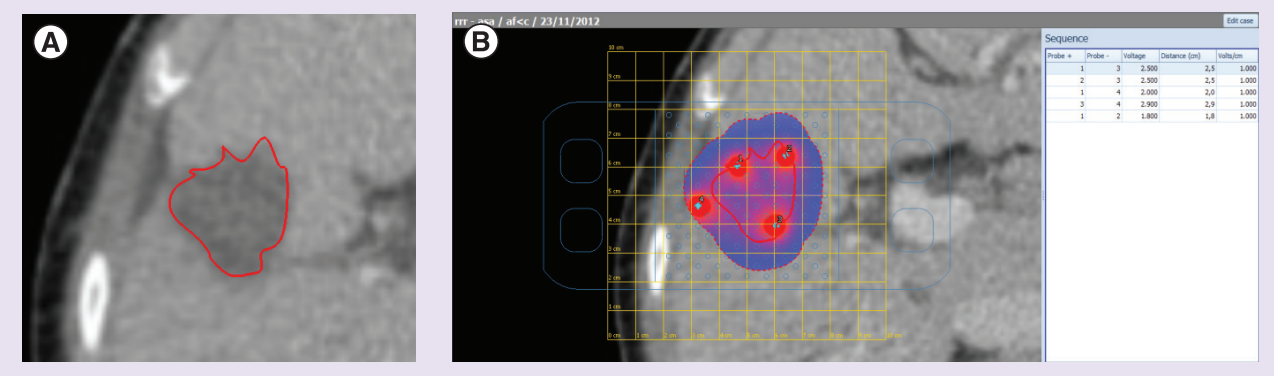

Figure 2. Preoperative planning software. (A) The red line indicates margins of a tumor lesion at the liver. (B) The preoperative planning software indicates where to position the electrodes (green dots within superimposed grid) and the electroporated area (blue). Planning software provides a table (far right) that indicates the electroporation pulse conditions to be applied to each couple of electrodes to obtain complete target tissue electroporation. 
on patients' survival and ultimately develop appropriate operating procedures.

\section{ECT in primary tumors}

The use of ECT for the treatment of primary tumors is at its early stages and it has been considered only for few well defined histotypes and localizations that currently represent unmet clinical needs.

Among skin tumors, while primary melanoma elective treatment is surgery, primary basal cell carcinoma and squamous cell carcinoma have been considered for ECT owing to limited tendency to metastasize. Complete response of basal cell carcinoma and squamous cell carcinoma is usually achieved after a single ECT session, recurrence is rare and the surgical option is always available. Basal cell carcinoma and squamous cell carcinoma treatment with ECT is particularly indicated in elderly patients and head and neck localizations. Gargiulo et al. has proposed ECT as the first line of treatment for head and neck tumors in consideration of the limited side effects, preservation of organ function and lack of impact on a surgical solutions if decided at a later stage [101].

ECT has been used in two independent earlyphase clinical trials for the treatment of primary breast cancer at the time of diagnosis in a neoadjuvant approach before tumor removal. Histological analysis at the time of surgery has demonstrated that tumor necrosis was not complete in most cases. Locally, an important inflammatory response was present and surgeons complained of the presence of fibrosis that made reconstruction difficult, both studies are currently active, but not enrolling patients while data are further analyzed [Di Filippo F, Garbay J-R, Pers. Сомm.].

In Bologna, in March 2013, over 300 clinicians have gathered to share their experiences (Second International ECT Users' Meeting [110]). Early clinical results on the use of ECT for the treatment of visceral primary tumors were presented. Clinical investigation was oriented toward those tumors that are difficult to resect and are located near important functional structures, nerve and vessels. Preclinical investigations in a small animal model support the safety of ECT when applied to pancreas.

\section{Conclusion}

For many years, ECT use has been limited to few oncological centers and mostly for research purposes, although efficacy, ease of use and lack of negative side effects were already well documented. Only after the completion of the two European projects Cliniporator and ESOPE, the bases were set for the dissemination of the treatment and its use in the clinical practice; since 2006, clear indications for use and precise definition of the SOPs have been available.

It is established that ECT is an effective method of treatment of neoplastic lesions located in the skin or subcutaneous tissue, both primary and metastatic, regardless of the histological type of the neoplasm or previous treatments. ECT established indications include: in-transit melanoma metastasis, basal cell carcinoma, squamous cell carcinoma, Kaposi sarcoma, skin metastases and chest wall recurrences from breast cancer, and head and neck tumors. ECT is an efficacious treatment method in patients with unresectable lesions, or those in whom standard treatment options failed or their application is not possible. It should also be noted that ECT is well accepted by patients, as indicated by the high percentage of those who would accept retreatment, if necessary [44,57].

Controlled trials demonstrated that ECT statistically and significantly increased the percentage of nodules attaining response to treatment or local tumor control in comparison with cytostatic agents (bleomycin or cisplatin) used in monotherapy. There was a large and clinically significant difference in the strength of intervention between ECT and monotherapy with a cytostatic agent. A high response to treatment rate (approximately 90\%) accompanied by a low local relapse rate $(10.6 \%)$ may be achieved after one ECT procedure. In addition, a positive effect of ECT on the patients' quality of life was demonstrated.

ECT is usually applied in palliative settings in treatment of patients with unresectable tumors and resulting in amelioration of quality of life. In most cases, it is used in treatment of advanced neoplastic lesions in which radical surgical treatment is not possible (e.g., due to lesion location, size and/or number). The objective of ECT is to improve local control of the disease and healthrelated quality of life, which is very important from the patient's point of view. Data available at present do not support an impact of ECT on overall survival.

ECT allows to treat tumor nodules in the proximity of important structures like vessels and nerves. The safety profile of ECT is favorable. Most of the observed adverse events are local and transient, including moderate local 
pain, erythema, edema and muscle contractions during EP. No serious adverse events or deaths related to ECT have been reported.

\section{Future perspective}

The medical community has clearly recognized the importance of ECT treatment and its advantages for patients, that in most instances would be lacking in other viable therapeutic alternatives. Ultimately, cell membrane EP must be viewed as a technique that locally enhances chemotherapy. It has been observed that following systemic chemotherapy metastases to the skin are often the sole remaining manifestation of disease; as such, we think that ECT might be included in the treatment protocols offered by the medical oncologist to obtain complete remission. The importance of reaching complete remission from disease in patients otherwise responding to systemic chemotherapy, or other interventions, should be highlighted and discussed among those determining what options are ultimately offered to cancer patients.

Following ECT, when cells enter mitosis, they die as a result of nonreparable DNA damage caused by bleomycin or cisplatin activity; however, as ECT does not induce protein denaturation, tumor-specific antigens, if present, may be released intact and recognized by inflammatory cells that migrate to the nodule. We already referred of the observation by Mir et al. that in nude mice ECT is less effective [22]. Furthermore, as proof of principle, the same group reported that in mice transplanted with two tumor nodules at two different sites, the treatment of one tumor nodule with ECT, when associated to stimulation of mice immune system with CpGs, induced the healing of the distant untreated nodule [111].

In a clinical series, Gehl et al. reported that the treatment of melanoma metastatic nodules with ECT and IL-2 resulted in a long-lasting remission of distant metastasis in $20 \%$ of

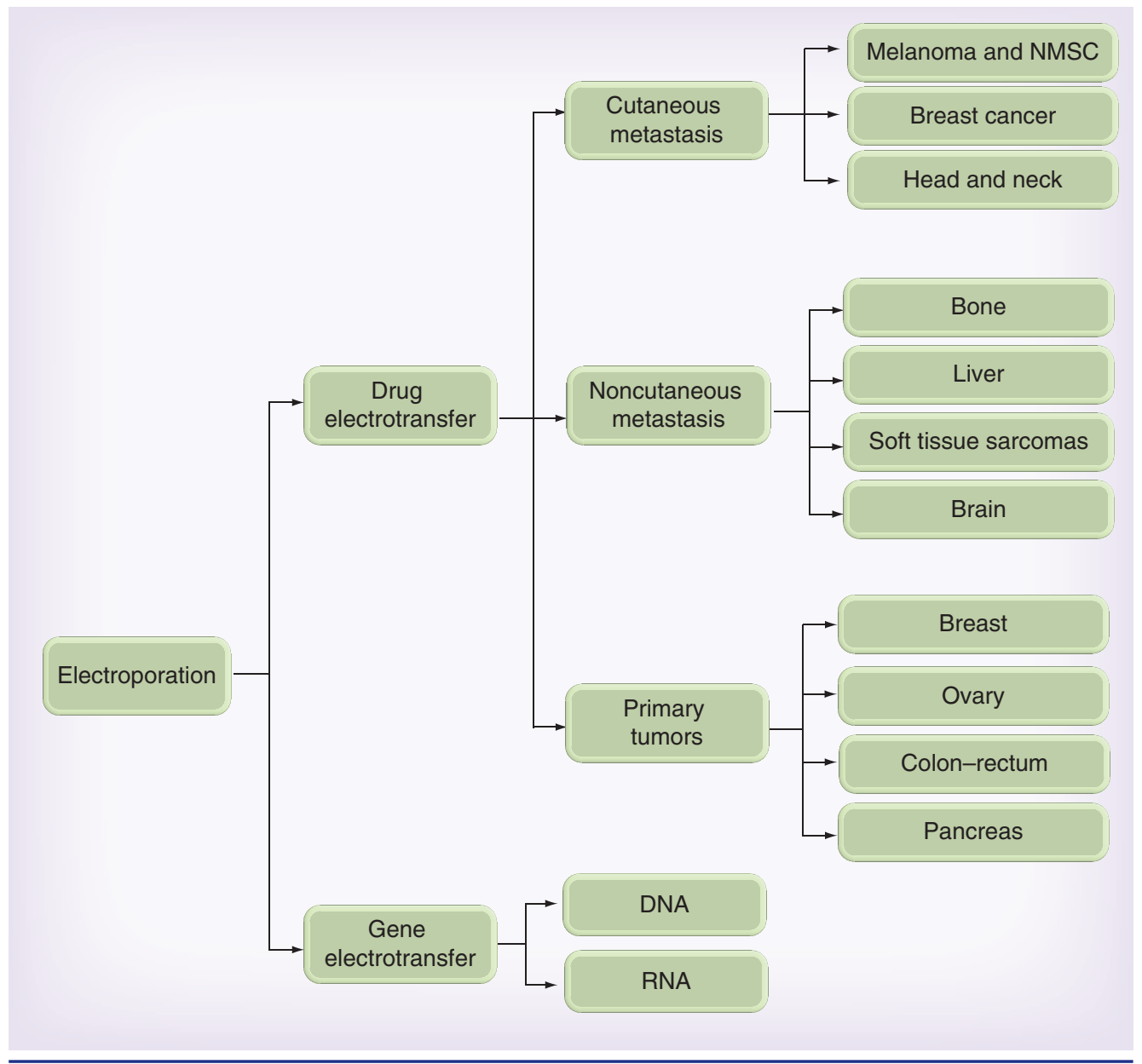

Figure 3. Perspective clinical applications of electroporation.

NMSC: Nonmelanoma skin cancer. 
patients [82]. Based on these observations, it is important to understand whether, and how, the local response triggered by ECT may be used to elicit a systemic response. To this aim, investigation by Gerlini and colleagues has focused on the timing of the sensitization of antigenpresenting cells to tumor antigens at the site of treatment. These findings would enable identification of the appropriate time to pharmacologically boost the immune system. Gerlini et al. conducted repeated histological tests at ECT melanoma-treated nodules demonstrating that at day 7 after treatment, there are no Langerhans cells in the nodule, while at day 14 , activated cells are back into the nodule [27].

The idea at the foundation of ECT is simple and effective and the medical community is now focused on the investigation of new perspectives of use for ECT and for EP in gene electrotransfer [112-114]. EP is a physical technique that provides

\section{EXECUTIVE SUMMARY}

\section{Electrochemotherapy}

- Electrochemotherapy (ECT) is the result of a combined treatment associating chemotherapeutic drugs and local application of electric pulses to induce cell membrane electroporation (EP). In vitro bleomycin cytotoxicity increased up to 1000 -fold and that of cisplatin by 80 -fold. Subsequently, prospective randomized studies (drug vs drug plus EP) clearly showed that the combined treatment was more effective than the two components used separately in the treatment of metastatic tumor nodules in patients.

\section{Development of standard operating procedures}

- The international multicenter clinical study ESOPE developed the standards of operating procedures for ECT performed with the Cliniporator ${ }^{\mathrm{TM}}$ (IGEA, SpA, Italy), which has allowed the dissemination and adoption of the therapy in over 120 cancer centers in Europe.

\section{Clinical experiences}

- ECT is used mainly in treatment of superficial (i.e., skin and subcutaneous) neoplastic lesions regardless of their histology. Review of studies involving 1894 tumors demonstrate that objective nodule objective response rate to ECT is $84 \%$. ECT indications include metastatic melanoma, Kaposi sarcoma, basal cell carcinoma, squamous cell carcinoma, breast cancer metastases to the skin, and head and neck tumors.

\section{ECT safety profile}

- The safety profile of ECT is favorable, with a limited number of adverse events reported, mostly moderate and transient.

\section{Treatment of nonsuperficial metastases}

- Bone and liver metastases have been treated with ECT in Phase II and Phase I/II clinical trials, respectively, showing treatment safety and activity.

\section{ECT in primary tumors}

- ECT has been used in skin primary tumors with success. ECT is being considered for the treatment of head and neck primary tumors.

\section{ECT \& immune response}

- The capability to trigger a systemic response after ECT by stimulating the immune system has been demonstrated in preclinical research. The activation of antigen-presenting cells at the site of treatment is presently under investigation.

\section{Conclusion}

- ECT is an effective treatment of neoplastic lesions located in the skin or subcutaneous tissue, both primary and metastatic, regardless of the histological type of the neoplasm or previous treatments. No serious adverse events or severe toxicity have been related to ECT.

\section{Future perspective}

- EP phenomenon provides the foundation not only for ECT, but for several treatment options, including gene therapy and DNA vaccination. EP technology shows the characteristics of a platform technology. 
the foundation for several treatment options and shows all characteristics of a platform technology with multiple clinical applications at different stages of development (Figure 3). Continuous technological development and clinical investigation of EP-based treatments will further increase clinical relevance of the technology.

Financial \& competing interests disclosure

$R$ Cadossi is a founder of IGEA. M Ronchetti is an employee of IGEA. IGEA manufactures a device for electrochemotherapy. The authors have no other relevant affiliations or

\section{References}

Papers of special note have been highlighted as:

- of interest; $\bullet$ of considerable interest

1 Orlowski S, Belehradek J Jr, Paoletti C, Mir LM. Transient electropermeabilization of cells in culture. Increase of the cytotoxicity of anticancer drugs. Biochem. Pharmacol. 37(24), 4727-4733 (1988).

-• First in vitro demonstration that cell membrane electroporation increases cytotoxicity of low permeant drugs.

2 Neumann E, Kakorin S, Toensing K. Fundamentals of electroporative delivery of drugs and genes. Bioelectrochem. Bioenerg. 48(1), 3-16 (1999).

3 Teissie J, Golzio M, Rols MP. Mechanisms of cell membrane electropermeabilization: a minireview of our present (lack of ?) knowledge. Biochim. Biophys. Acta 1724(3), 270-280 (2005).

4 Kramar P, Delemotte L, Maček Lebar A, Kotulska M, Tarek M, Miklavčič D. Molecular-level characterization of lipid membrane electroporation using linearly rising current. J. Membr. Biol. 245(10), 651-659 (2012).

5 Neumann E, Schaefer-Ridder M, Wang Y, Hofschneider PH. Gene transfer into mouse lyoma cells by electroporation in high electric fields. EMBO J. 1(7), 841-845 (1982).

6 Mir LM, Orlowski S. The basis of electrochemotherapy. Methods Mol. Med. 37, 99-117 (2000).

7 Rols MP, Golzio M, Gabriel B, Teissié J. Factors controlling electropermeabilisation of cell membranes. Technol. Cancer Res. Treat 1(5), 319-328 (2002).

8 Mesojednik S, Pavlin D, Sersa G et al. The effect of the histological properties of tumors on transfection efficiency of electrically assisted gene delivery to solid tumors in mice. Gene Ther. 14(17), 1261-1269 (2007).

financial involvement with any organization or entity with a financial interest in or financial conflict with the subject matter or materials discussed in the manuscript apart from those disclosed.

No writing assistance was utilized in the production of this manuscript.

\section{Open Access}

This work is licensed under the Creative Commons Attribution-NonCommercial 3.0 Unported License. To view a copy of this license, visit http://creativecommons.org/ licenses/by-nc-nd/3.0/

9 Fini $\mathrm{M}$, Tschon $\mathrm{M}$, Ronchetti $\mathrm{M}$ et al. Ablation of bone cells by electroporation. J. Bone Joint Surg. Br. 92(11), 1614-1620 (2010).

10 Gehl J, Skovsgaard T, Mir LM. Enhancement of cytotoxicity by electropermeabilization: an improved method for screening drugs. Anticancer Drugs 9(4), 319-325 (1998).

11 Ongaro A, Pellati A, Caruso A et al. Identification of in vitro electropermeabilization equivalent pulse protocols. Technol. Cancer Res. Treat. 10(5), 465-473 (2011).

12 Mir LM, Orlowski S, Belehradek J Jr, Paoletti C. Electrochemotherapy potentiation of antitumour effect of bleomycin by local electric pulses. Eur. J. Cancer 27(1), 68-72 (1991).

13 Belehradek J Jr, Orlowski S, Poddevin B, Paoletti C, Mir LM. Electrochemotherapy of spontaneous mammary tumours in mice. Eur. J. Cancer 27(1), 73-76 (1991).

14 Sersa G, Cemazar M, Miklavcic D. Antitumor effectiveness of electrochemotherapy with cisdiamminedichloroplatinum(II) in mice. Cancer Res. 55(15), 3450-3455 (1995).

15 Orlowski S, Mir LM. Treatment of multiple spontaneous breast tumors in mice using electrochemotherapy. Methods Mol. Med. 37, 265-269 (2000).

16 Cemazar M, Miklavcic D, Mir LM et al. Electrochemotherapy of tumours resistant to cisplatin: a study in a murine tumour model. Eur. J. Cancer 37(9), 1166-1172 (2001).

17 Lebar AM, Sersa G, Kranjc S, Groselj A, Miklavcic D. Optimisation of pulse parameters in vitro for in vivo electrochemotherapy. Anticancer Res. 22(3), 1731-1736 (2002).

18 Sersa G, Kranjc S, Scancar J, Krzan M, Cemazar M. Electrochemotherapy of mouse sarcoma tumors using electric pulse trains with repetition frequencies of $1 \mathrm{~Hz}$ and $5 \mathrm{kHz}$. J. Membr. Biol. 236(1), 155-162 (2010).
19 Fini M, Salamanna F, Parrilli A et al. Electrochemotherapy is effective in the treatment of rat bone metastases. Clin. Exp. Metastasis 30 (8), 1033-1045 (2013).

20 Agerholm-Larsen B, Iversen HK, Ibsen P et al. Preclinical validation of electrochemotherapy as an effective treatment for brain tumors. Cancer Res. 71(11), 3753-3762 (2011).

21 Linnert M, Iversen HK, Gehl J. Multiple brain metastases - current management and perspectives for treatment with electrochemotherapy. Radiol. Oncol. 46(4), 271-278 (2012).

22 Sersa G, Miklavcic D, Cemazar M, Belehradek J Jr, Jarm T, Mir LM. Electrochemotherapy with CDDP on LPB sarcoma: comparison of the anti-tumor effectiveness in immunocompetent and immunodeficient mice. Bioelectrochem. Bioenerg. 43, 279-283 (1997).

23 Sersa G, Kotnik V, Cemazar M, Miklavcic D, Kotnik A. Electrochemotherapy with bleomycin in SA-1 tumor-bearing mice - natural resistance and immune responsiveness. Anticancer Drugs 7(7), 785-791 (1996).

24 Mir LM, Orlowski S, Poddevin B, Belehradek $\mathrm{J} \mathrm{Jr}$. Electrochemotherapy tumor treatment is improved by interleukin-2 stimulation of the host's defenses. Eur. Cytokine Netw. 3(3), 331-334 (1992).

-. Demonstrates the contribution of the immune system to electrochemotherapy treatment.

25 Zheng MH, Feng B, Li JW et al. Effects and possible anti-tumor immunity of electrochemotherapy with bleomycin on human colon cancer xenografts in nude mice. World J. Gastroenterol. 11(16), 2426-2430 (2005).

26 Gerlini G, Di Gennaro P, Borgognoni L. Enhancing anti-melanoma immunity by electrochemotherapy and in vivo dendriticcell activation. Oncoimmunology 1(9), 1655-1657 (2012). 
27 Gerlini G, Sestini S, Di Gennaro P, Urso C, Pimpinelli N, Borgognoni L. Dendritic cells recruitment in melanoma metastasis treated by electrochemotherapy. Clin. Exp. Metastasis 30(1), 37-45 (2013).

28 Belehradek M, Domenge C, Luboinski B, Orlowski S, Belehradek J Jr, Mir LM. Electrochemotherapy, a new antitumor treatment. First clinical Phase I-II trial. Cancer 72(12), 3694-3700 (1993).

29 Glass LF, Jaroszeski M, Gilbert R, Reintgen DS, Heller R. Intralesional bleomycinmediated electrochemotherapy in 20 patients with basal cell carcinoma. J. Am. Acad. Dermatol. 37(4), 596-599 (1997).

30 Sersa G, Stabuc B, Cemazar M, Jancar B, Miklavcic D, Rudolf Z. Electrochemotherapy with cisplatin: potentiation of local cisplatin antitumour effectiveness by application of electric pulses in cancer patients. Eur. J. Cancer 34(8), 1213-1218 (1998).

31 Glass LF, Pepine ML, Fenske NA, Jaroszeski M, Reintgen DS, Heller R. Bleomycinmediated electrochemotherapy of metastatic melanoma. Arch. Dermatol. 132(11), 1353-1357 (1996).

32 Heller R, Jaroszeski MJ, Reintgen DS et al. Treatment of cutaneous and subcutaneous tumors with electrochemotherapy using intralesional bleomycin. Cancer 83(1), 148-157 (1998).

33 Sersa G, Stabuc B, Cemazar M, Miklavcic D, Rudolf Z. Electrochemotherapy with cisplatin: clinical experience in malignant melanoma patients. Clin. Cancer Res. 6(3), 863-867 (2000).

34 Byrne CM, Thompson JF, Johnston $\mathrm{H}$ et al. Treatment of metastatic melanoma using electroporation therapy with bleomycin (electrochemotherapy). Melanoma Res. 15(1), 45-51 (2005).

35 Gaudy C, Richard MA, Folchetti G, Bonerandi JJ, Grob JJ. Randomized controlled study of electrochemotherapy in the local treatment of skin metastases of melanoma. J. Cutan. Med. Surg. 10(3), 115-121 (2006).

36 Miller AB, Hoogstraten B, Staquet M, Winkler A. Reporting results of cancer treatment. Cancer 47, 207-214 (1981).

37 Quaglino P, Mortera C, Osella-Abate S et al. Electrochemotherapy with intravenous bleomycin in the local treatment of skin melanoma metastases. Ann. Surg. Oncol. 15(8), 2215-2222 (2008).

38 Quaglino P, Mortera C, Marenco F. Electrochemotherapy in the treatment of cutaneous melanoma metastases: long-term follow-up results and response-associated clinic-pathologic factors. Madrid 51, Abstract 05 (2010).

39 Mir LM, Glass LF, Sersa G et al. Effective treatment of cutaneous and subcutaneous malignant tumours by electrochemotherapy. Br. J. Cancer 77(12), 2336-2342 (1998).

40 Heller R, Gilbert R, Jaroszeski MJ. Clinical applications of electrochemotherapy. $A d v$. Drug Deliv. Rev. 35(1), 119-129 (1999).

41 Gothelf A, Mir LM, Gehl J. Electrochemotherapy: results of cancer treatment using enhanced delivery of bleomycin by electroporation. Cancer Treat. Rev. 29(5), 371-387 (2003).

42 Mir LM, Morsli N, Garbay JR, Billard V, Robert C, Marty M. Electrochemotherapy: a new treatment of solid tumors. J. Exp. Clin. Cancer Res. 22(4 Suppl.), 145-148 (2003).

43 Larkin JO, Collins CG, Aarons S et al. Electrochemotherapy: aspects of preclinical development and early clinical experience. Ann. Surg. 245(3), 469-479 (2007).

44 Marty M, Sersa G, Garbay JR et al. Electrochemotherapy - an easy, highly effective and safe treatment of cutaneous and subcutaneous metastases: results of ESOPE (European Standard Operating Procedures of Electrochemotherapy) study. EJC Suppl. 4(11), 3-13 (2006).

- Demonstrates, in a multicenter study, the efficacy of electrochemotherapy with bleomycin and cisplatin and the equivalence of intravenously and intratumoral drug injection.

45 Mir LM, Gehl J, Sersa G et al. Standard operating procedures of the electrochemotherapy: instructions for use of bleomycin or cisplatin administered either systemically or locally and electric pulse delivered by the Cliniporator by means of invasive and non-invasive electrodes. EJC Suppl. 4(11), 14-25 (2006).

- Standard operating procedures for use in daily clinical practice are described.

46 Kendler M, Micheluzzi M, Wetzig T, Simon JC. Electrochemotherapy under tumescent local anesthesia for the treatment of cutaneous metastases. Dermatol. Surg. 39(7), 1023-1032 (2013).

47 Mali B, Jarm T, Snoj M, Sersa G, Miklavcic D. Antitumor effectiveness of electrochemotherapy: a systematic review and meta-analysis. Eur. J. Surg. Oncol. 39(1), 4-16 (2012).

-• First meta-analysis of clinical studies reported in the literature.
48 Matthiessen LW, Chalmers RL, Sainsbury DC et al. Management of cutaneous metastases using electrochemotherapy. Acta Oncol. 50(5), 621-629 (2011).

49 Electrochemotherapy for the treatment of malignant melanoma.

http://guidance.nice.org.uk/IP/1041

50 Electrochemotherapy for primary basal cell carcinoma and primary squamous cell carcinoma (IPG447). http://guidance.nice.org.uk/IP/1017

51 Electrochemotherapy for metastases in the skin from tumours of non-skin origin (IPG446). http://guidance.nice.org.uk/IP/984

52 Giardino R, Fini M, Bonazzi V, Cadossi R, Nicolini A, Carpi A. Electrochemotherapy a novel approach to the treatment of metastatic nodules on the skin and subcutaneous tissues. Biomed. Pharmacother. 60 (8), 458-462 (2006).

53 Reinhold U. Electrochemotherapy of skin tumors. Hautarzt 62 (7), 549-558 (2011).

54 Skarlatos I, Kyrgias G, Mosa E et al. Electrochemotherapy in cancer patients: first clinical trial in Greece. In Vivo 25(2), 265-274 (2011).

55 Muñoz Madero V, Ortega Pérez G. Electrochemotherapy for treatment of skin and soft tissue tumours. Update and definition of its role in multimodal therapy. Clin. Transl. Oncol. 13(1), 18-24 (2011).

56 Testori A, Tosti G, Martinoli C et al. Electrochemotherapy for cutaneous and subcutaneous tumor lesions: a novel therapeutic approach. Dermatol. Ther. 23(6), 651-661 (2010).

- Describes the indications for use of electrochemotherapy in clinical practice.

57 Campana LG, Mocellin S, Basso M et al. Bleomycin-based electrochemotherapy: clinical outcome from a single institution's experience with 52 patients. Ann. Surg. Oncol. 16(1), 191-199 (2009).

58 Carrera C, Bennassar A, Ishioka P et al. Desmoplastic melanoma on the nose: electrochemotherapy as an alternative treatment to local advanced disease. J. Eur. Acad. Dermatol. Venereol. doi:10.1111/ jdv.12115 (2013) (Epub ahead of print).

59 Wiater K, Zdzienicki M, Morysi ski T. Effective treatment of recurrent, advanced dermatofibrosarcoma protuberans by electrochemotherapy. Eur. J. Dermatol. 23(2), 260-261 (2013).

60 Curatolo P, Rotunno R, Miraglia E, Mancini M, Calvieri S, Giustini S. Complete remission of Merkel cell carcinoma treated with 
electrochemotherapy and etoposide. G. Ital. Dermatol. Venereol. 148(3), 310-311 (2013).

Gehl J, Geertsen PF. Efficient palliation of haemorrhaging malignant melanoma skin metastases by electrochemotherapy. Melanoma Res. 10(6), 585-589 (2000).

62 Jarm T, Cemazar M, Miklavcic D, Sersa G. Antivascular effects of electrochemotherapy: implications in treatment of bleeding metastases. Expert Rev. Anticancer Ther. 10(5), 729-746 (2010).

63 Benevento R, Santoriello A, Perna G, Canonico $S$. Electrochemotherapy of cutaneous metastastes from breast cancer in elderly patients: a preliminary report. $B M C$ Surg. 12(Suppl. 1), S6 (2012).

64 Mali B, Miklavcic D, Campana LG et al. Tumor size and effectiveness of ECT. Radiol. Oncol. 47(1), 32-41 (2013).

65 Kaehler KC, Egberts F, Hauschild A. Electrochemotherapy in symptomatic melanoma skin metastases: intraindividual comparison with conventional surgery. Dermatol. Surg. 36(7), 1200-1202 (2010).

66 Snoj M, Cemazar M, Srnovrsnik T, Kosir SP, Sersa G. Limb sparing treatment of bleeding melanoma recurrence by electrochemotherapy. Tumori 95(3), 398-402 (2009).

67 Gehl J, Geertsen PF. Palliation of haemorrhaging and ulcerated cutaneous tumors using electrochemotherapy. EJC Suppl. 4, 35-37(2006).

68 Whelan MC, Larkin JO, Collins CG et al. Effective treatment of an extensive recurrent breast cancer which was refractory to multimodal therapy by multiple applications of electrochemotherapy. EJC Suppl. 4(11), 32-34 (2006).

69 Fantini F, Gualdi G, Cimitan A, Giannetti A. Metastatic basal cell carcinoma with squamous differentiation: report of a case with response of cutaneous metastases to electrochemotherapy. Arch. Dermatol. 144(9), 1186-1188 (2008).

70 Tassinari J, Orlandino G, Ardito R. Electrochemotherapy in oncology reconstructive surgery: five cases preliminary report. Riv. ltal. Chir. Plastica 40, 55-60 (2008).

71 Gualdi G, Monari P, Fantini F, Cesinaro AM, Cimitan A. Electrochemotherapy-induced virus disappearance in HHV-8-positive skin nodules of Kaposi sarcoma: first histological and immunohistochemical demonstration of efficacy. J. Eur. Acad. Dermatol. Venereol. 24(2), 239-241 (2010).

72 Richetta AG, Curatolo P, D’Epiro S et al. Efficacy of electrochemotherapy in ulcerated basal cell carcinoma. Clin. Ter. 162(5), 443-445 (2011).

73 Mozzillo N, Caracò C, Mori S et al. Use of neoadjuvant electrochemotherapy to treat a large metastatic lesion of the cheek in a patient with melanoma. J. Transl Med. 10, 131 (2012).

74 Tauceri F, Mura G, Roseano M, Framarini M, Ridolfi L, Verdecchia GM. Surgery and adjuvant therapies in the treatment of stage IV melanoma: our experience in 84 patients. Langenbecks Arch. Surg. 394(6), 1079-1084 (2009).

75 Kis E, Oláh J, Ócsai H et al.

Electrochemotherapy of cutaneous metastases of melanoma - a case series study and systematic review of the evidence. Dermatol. Surg. 37(6), 816-824 (2011).

76 Testori A, Intelisano A, Verrecchia F et al. Alternatives for the treatment of local advanced disease: electrochemotherapy, limb perfusion, limb infusion, intralesional IL2. What is the role? Dermatol. Ther. 25(5), 443-451 (2012).

77 Campana LG, Valpione S, Mocellin S et al. Electrochemotherapy for disseminated superficial metastases from malignant melanoma. Br. J. Surg. 99(6), 821-830 (2012).

78 Testori A, Rossi CR, Tosti G. Utility of electrochemotherapy in melanoma treatment. Curr. Opin Oncol. 24(2), 155-161 (2012).

79 Byrne CM, Thompson JF. Role of electrochemotherapy in the treatment of metastatic melanoma and other metastatic and primary skin tumors. Expert Rev. Anticancer Ther. 6(5), 671-678 (2006).

80 Testori A, Soteldo J, Powell B et al. Surgical management of melanoma: an EORTC Melanoma Group survey. Ecancermedicalscience 7, 294 (2013).

81 Rastrelli M, Alaibac M, Stramare R et al. Melanoma $\mathrm{m}$ (zero): diagnosis and therapy. ISRN Dermatol., 616170 (2013).

82 Gehl J, Andersen MH, Hastrup N, Straten PT, Geertsen PF. Electrochemotherapy with low-dose IL-2 in the treatment of disseminated malignant melanoma: clinical and paraclinical evidence of systemic immune response. Proc. Am. Soc. Clin. Oncol. 22, Abstract 2906 (2003).

83 Simeone E, Benedetto L, Gentilcore G et al. Combination therapy with ipilimumab and electrochemotherapy: preliminary efficacy results and correlation with immunologic parameters. J. Clin. Oncol. 31(Suppl.) Abstract e20031 (2013).

84 Garbay JR, Billard V, Bernat C, Mir LM, Morsli N, Robert C. Successful repetitive treatments by electrochemotherapy of multiple unresectable Kaposi sarcoma nodules. EJC Suppl. 4, 29-31 (2006).

85 Curatolo P, Quaglino P, Marenco F et al. Electrochemotherapy in the treatment of Kaposi sarcoma cutaneous lesions: a two-center prospective Phase II trial. Ann. Surg. Oncol. 19(1), 192-198 (2012).

86 Latini A, Bonadies A, Trento E et al. Effective treatment of Kaposi's sarcoma by electrochemotherapy and intravenous bleomycin administration. Dermatol. Ther 25(2), 214-218 (2012).

87 Di Monta G, Caracò C, Benedetto L et al. Electrochemotherapy as 'new standard of care' treatment for cutaneous Kaposi's sarcoma. Eur. J. Surg. Oncol. 40 (1), 61-66 (2013).

88 Rodríguez-Cuevas S, Barroso-Bravo S, Almanza-Estrada J, Cristóbal-Martínez L, González-Rodríguez E. Electrochemotherapy in primary and metastatic skin tumors: Phase II trial using intralesional bleomycin. Arch. Med. Res. 32(4), 273-276 (2001).

89 Kis E, Baltás E, Kinyó A et al. Successful treatment of multiple basaliomas with bleomycin-based electrochemotherapy: a case series of three patients with Gorlin-Goltz syndrome. Acta Derm. Venereol. 92(6), 648-651 (2012).

90 Salwa SP, Bourke MG, Forde PF et al. Electrochemotherapy for the treatment of ocular basal cell carcinoma; a novel adjunct in the disease management. J. Plast. Reconstr. Aesthet. Surg. doi:10.1016/j.bjps.2013.07.019 (2013) (Epub ahead of print).

91 Perrone AM, Galuppi A, Cima S et al. Electrochemotherapy can be used as palliative treatment in patients with repeated loco-regional recurrence of squamous vulvar cancer: a preliminary study. Gynecol. Oncol. 130(3), 550-553 (2013).

92 Campana LG, Valpione S, Falci C. The activity and safety of electrochemotherapy in persistent chest wall recurrence from breast cancer after mastectomy: a Phase-II study. Breast Cancer Res. Treat. 134(3), 1169-1178 (2012).

-• Reports a clinical experience in the treatment of breast cancer metastases to the skin, demonstrating the importance of early treatment with electrochemotherapy.

93 Matthiessen LW, Johannesen HH, Hendel HW, Moss T, Kamby C, Gehl J. Electrochemotherapy for large cutaneous recurrence of breast cancer: a Phase II clinical trial. Acta Oncol. 51(6), 713-721 (2012). 
94 Sersa G, Cufer T, Paulin SM, Cemazar M, Snoj M. Electrochemotherapy of chest wall breast cancer recurrence. Cancer Treat. Rev . 38(5), 379-386 (2012).

95 Burian M, Formanek M, Regele H. Electroporation therapy in head and neck cancer. Acta Otolaryngol. 123, 264-268 (2003).

96 Landström FJ, Nilsson CO, Crafoord S, Reizenstein JA, Adamsson GB, Löfgren LA. Electroporation therapy of skin cancer in the head and neck area. Dermatol. Surg. 36(8), 1245-1250 (2010).

97 Landstrom FJ, Nilsson CO, Reizenstein JA, Nordqvist K, Adamsson GB, Löfgren AL. Electroporation therapy for $\mathrm{T} 1$ and $\mathrm{T} 2$ oral tongue cancer. Acta Otolaryngol. 131(6), 660-664 (2011).

98 Allegretti JP, Panje WR. Electroporation therapy for head and neck cancer including carotid artery involvement. Laryngoscope 111(1), 52-56 (2001).

99 Kyrgias G, Kostopoulou E, Zafiriou E et al. Hidradenocarcinoma of the temporal area successfully treated with concomitant electrochemotherapy and radiotherapy. Head Neck Oncol. 5(2), 14, 2013.

100 Mevio N, Bertino G, Occhini A et al. Electrochemotherapy for the treatment of recurrent head and neck cancers: preliminary results. Tumori 98(3), 308-313 (2012).

- Defines the indications for use of electrochemotherapy in head and neck cancer patients.
101 Gargiulo M, Papa A, Capasso P, Moio M, Cubicciotti E, Parascandolo $S$.

Electrochemotherapy for non-melanoma head and neck cancers: clinical outcomes in 25 patients. Ann. Surg. 255(6), 1158-1164 (2012).

102 Inovio Biomedical Stops Enrollment of Phase III Clinical Trials for Head \& Neck Cancer. www.drugs.com/news/inovio-biomedicalstops-enrollment-phase-iii-clinical-trials-headamp-neck-cancer-6269.html

103 Kos B, Zupanic A, Kotnik T, Snoj M, Sersa G, Miklavcic D. Robustness of treatment planning for electrochemotherapy of deep-seated tumors. J. Membr. Biol. 236(1), 147-153 (2010).

104 Pavliha D, Kos B, Zupani A, Mar an M, Serša G, Miklav i D. Patient-specific treatment planning of electrochemotherapy: procedure design and possible pitfalls. Bioelectrochemistry 87, 265-273 (2012).

105 Bertacchini C, Margotti PM, Bergamini E, Lodi A, Ronchetti M, Cadossi R. Design of an irreversible electroporation system for clinical use. Technol. Cancer Res. Treat. 6(4), 313-320 (2007).

106 Mali B, Jarm T, Corovic S et al. The effect of electroporation pulses on functioning of the heart. Med. Biol. Eng. Comput. 46(8), 745-757 (2008).

107 Miklavi D, Serša G, Brecelj E et al. Electrochemotherapy: technological advancements for efficient electroporationbased treatment of internal tumors. Med.
Biol. Eng. Comput. 50(12), 1213-1225 (2012).

108 Bianchi G, Campanacci L, Fini M. Electrochemotherapy for the treatment of osteolytic bone metastasis: a Phase II clinical trial. Presented at: European Musculo-Skeletal Oncology Society. Bologna, Italy, 15-16 May 2010.

109 Edhemovic I, Gadzijev EM, Brecelj E et al. Electrochemotherapy: a new technological approach in treatment of metastases in the liver. Technol. Cancer Res. Treat. 10(5), 475-485 (2011).

110 Electrochemotherapy: 2nd International Users' Meeting, Come, Share, Learn. www.igeamedical.com/sites/default/files/ news_events_press/files_allegati/ igeameetingbo.pdf

111 Roux S, Bernat C, Al-Sakere B et al. Tumor destruction using electrochemotherapy followed by $\mathrm{CpG}$ oligodeoxynucleotide injection induces distant tumor responses. Cancer Immunol. Immunother. 57(9), 1291-1300 (2008).

112 Mir LM. Application of electroporation gene therapy: past, current, and future. Methods Mol. Biol. 423, 3-17 (2008).

113 Gehl J. Electroporation for drug and gene delivery in the clinic: doctors go electric. Methods Mol. Biol. 423, 351-359 (2008).

114 Andre FM, Mir LM. Nucleic acids electrotransfer in vivo: mechanisms and practical aspects. Curr. Gene Ther. 10(4), 267-280 (2010). 